\title{
On Types of Distance Fibonacci Numbers Generated by Number Decompositions
}

\author{
Anetta Szynal-Liana, Andrzej Włoch, and Iwona Włoch \\ Faculty of Mathematics and Applied Physics, Rzeszow University of Technology, Al. Powstańców Warszawy 12, 35-959 Rzeszow, Poland \\ Correspondence should be addressed to Andrzej Włoch; awloch@prz.edu.pl
}

Received 2 June 2014; Revised 21 August 2014; Accepted 23 August 2014; Published 23 October 2014

Academic Editor: Ali R. Ashrafi

Copyright (C) 2014 Anetta Szynal-Liana et al. This is an open access article distributed under the Creative Commons Attribution License, which permits unrestricted use, distribution, and reproduction in any medium, provided the original work is properly cited.

We introduce new types of distance Fibonacci numbers which are closely related with number decompositions. Using special decompositions of the number $n$ we give a sequence of identities for them. Moreover, we give matrix generators for distance Fibonacci numbers and their direct formulas.

\section{Introduction}

The $n$th Fibonacci numbers $F_{n}$ are defined by recurrence relation $F_{n}=F_{n-1}+F_{n-2}, n \geq 2$ with the initial conditions $F_{0}=$ $F_{1}=1$. There are many generalizations of the Fibonacci numbers $F_{n}$ with respect to one or more parameters; see for example [1-3]. In [1] the distance Fibonacci numbers $F d^{(1)}(k, n)$ were introduced and studied. We recall this definition.

Let $k \geq 2, n \geq 0$ be integers. The distance Fibonacci numbers of the first kind $F d^{(1)}(k, n)$ are defined recursively in the following way:

$$
\begin{aligned}
F d^{(1)}(k, n)= & F d^{(1)}(k, n-k+1) \\
& +F d^{(1)}(k, n-k) \quad \text { for } n \geq k,
\end{aligned}
$$

and $F d^{(1)}(k, n)=1$ for $n=0, \ldots, k-1$.

We will call the numbers $F d^{(1)}(k, n)$ the distance Fibonacci numbers of the first kind. The number $F d^{(1)}(k, n)$ is closely related to the special quasi $k$-decomposition of the number $n$; see [1].

In this paper we define other three types of distance Fibonacci numbers which are also related to the special number decomposition. Moreover we shall show relations between all three types of distance Fibonacci numbers. Next we study their matrix generators and direct formulas.

\section{Distance Fibonacci Numbers}

$$
F d^{(2)}(k, n) \text { and } F d^{(3)}(k, n)
$$

In this section we introduce two kinds of distance Fibonacci numbers. Some relations between numbers $F d^{(i)}(k, n)$ for $i=$ $1,2,3$ will be studied.

Let $k \geq 2, n \geq 0$ be integers. We define the $n$th distance Fibonacci numbers of the second kind $F d^{(2)}(k, n)$ by the $k$ th order linear recurrence relation of the form

$$
\begin{aligned}
F d^{(2)}(k, n)= & F d^{(2)}(k, n-k+1) \\
& +F d^{(2)}(k, n-k) \quad \text { for } n \geq k,
\end{aligned}
$$

with the initial conditions

$$
\begin{aligned}
& F d^{(2)}(k, n)=0 \text { for } n=0, \ldots, k-2, \\
& F d^{(2)}(k, k-1)=1, \\
& F d^{(2)}(1,1)=1, \\
& F d^{(2)}(2,2)=2, \\
& F d^{(2)}(k, k)=1, \text { for } k \geq 3 .
\end{aligned}
$$

If $k=2, n \geq 1$, then $F d^{(2)}(k, n)$ gives the Fibonacci numbers $F_{n}$. 
Let $k \geq 2, n \geq 0$ be integers. We define the $n$th distance Fibonacci numbers of the third kind $\operatorname{Fd}^{(3)}(k, n)$ by the $k$ th order linear recurrence relation of the form

$$
\begin{aligned}
F d^{(3)}(k, n)= & F d^{(3)}(k, n-k+1) \\
& +F d^{(3)}(k, n-k) \quad \text { for } n \geq 2 k-1,
\end{aligned}
$$

with the initial conditions

$$
\begin{aligned}
& F d^{(3)}(k, n)=1 \text { for } n=0, \ldots, k-1, \\
& F d^{(3)}(2,2)=2,
\end{aligned}
$$$$
\text { for } k \geq 3 F d^{(3)}(k, k)=3=F d^{(3)}(k, 2 k-2) \text {, }
$$$$
\text { for } k+1 \leq n \leq 2 k-3 F d^{(3)}(k, n)=4 \text {. }
$$

If $k=2$, then $F d^{(3)}(k, n)$ gives the classical Fibonacci numbers.

Now we give an interpretation of the numbers $F d^{(1)}(k, n)$, $F d^{(2)}(k, n)$, and $F d^{(3)}(k, n)$ with respect to special decompositions of the number $n$.

By a decomposition of a number $n, n \geq 1$, we mean an ordered number partition of it. For example for $n=3$ we have the following four decompositions: $1+1+1,2+1,1+$ 2 , and 3. In this paper we study special decompositions of a number $n$ which are closely related to distance Fibonacci numbers $F d^{(i)}(k, n)$, for $i=1,2,3$.

Let $1 \leq r \leq k-2$ be a fixed integer. A decomposition of the number $n \geq k-1$ of the form $r+n_{1}+n_{2}+\cdots+n_{p}$ (resp., $\left.n_{1}+n_{2}+\cdots+n_{p}+r\right)$ where $n_{i} \in\{k, k-1\}, i=1, \ldots, p$ is called an $r_{-} k$-decomposition (resp., $r_{+} k$-decomposition). We denote the number of all $r_{-} k$-decompositions (resp., $r_{+} k$ decompositions) by $\sigma_{-r}(k, n)$ (resp., $\left.\sigma_{+r}(k, n)\right)$. Clearly

$$
\sigma_{-r}(k, n)=\sigma_{+r}(k, n)
$$

A decomposition of the number $n \geq k-1$ of the form $n_{1}+$ $n_{2}+\cdots+n_{p}$, where $n_{i} \in\{k, k-1\}, i=1, \ldots, p$, is called a $k$-decomposition. We denote the number of all $k$-decompositions by $\sigma_{0}(k, n)$.

Let $0 \leq r_{0} \leq k-2$ be a fixed integer. A decomposition of the number $n \geq k-1$ of the form $r_{0}+n_{1}+n_{2}+\cdots+$ $n_{p}$ (resp., $n_{1}+n_{2}+\cdots+n_{p}+r_{0}$ ) where $n_{i} \in\{k, k-1\}$, $i=1, \ldots, p$ is called an $r_{0-} k$-decomposition (resp., $r_{0+} k$ decomposition). Consequently as the above we denote the number of all $r_{0-} k$-decompositions (resp., $r_{0+} k$-decompositions) by $\sigma_{-r_{0}}(k, n)$ (resp., $\left.\sigma_{+r_{0}}(k, n)\right)$. Clearly for $r_{0}=0$ a $0_{-} k$-decomposition of $n$ is a $k$-decomposition and for $r_{0} \geq 1$ an $r_{0-} k$-decomposition (resp., $r_{0+} k$-decompositions) is an $r_{-} k$-decompositions (resp., $r_{+} k$-decompositions). From the above definitions immediately follow relations between numbers $\sigma_{-r}(k, n), \sigma_{-r_{0}}(k, n)$, and $\sigma_{0}(k, n)$ (resp., $\sigma_{+r}(k, n)$, $\sigma_{+r_{0}}(k, n)$, and $\left.\sigma_{0}(k, n)\right)$ :

$$
\begin{aligned}
& \sigma_{-r_{0}}(k, n)=\sum_{r=1}^{k-2} \sigma_{-r}(k, n)+\sigma_{0}(k, n), \\
& \sigma_{+r_{0}}(k, n)=\sum_{r=1}^{k-2} \sigma_{+r}(k, n)+\sigma_{0}(k, n),
\end{aligned}
$$

$$
\sigma(k, n)=\sum_{j=-(k-2)}^{k-2} \sigma_{j}(k, n) .
$$

Theorem 1 (see [1]). Let $k \geq 2, n \geq k-1$ be integers. Then $\sigma_{+r_{0}}(k, n)=F d^{(1)}(k, n)$.

For the proof of the next theorem we will need the following lemma.

Lemma 2. Let $k \geq 2, n \geq k-1$ be integers. Then

$$
\begin{array}{r}
2 F d^{(1)}(k, n)-F d^{(2)}(k, n)=F d^{(3)}(k, n), \\
\text { for } n \geq k-1 .
\end{array}
$$

Proof. If $n=k-1$, then the equality immediately follows. Assume that the lemma is true for an arbitrary $t<n$ and we prove it for $n$. Using the definitions of numbers $F d^{(1)}(k, n)$ and $F d^{(2)}(k, n)$ we obtain that $2 F d^{(1)}(k, n)-F d^{(2)}(k, n)=$ $2 F d^{(1)}(k, n-k)+2 F d^{(1)}(k, n-k+1)-F d^{(2)}(k, n-k)-F d^{(2)}(k, n-$ $k+1)=F d^{(3)}(k, n)$ by the induction's hypothesis.

We can write the above lemma also in the following form.

Corollary 3. Let $k \geq 2, n \geq k-1$ be integers. Then

$$
F d^{(1)}(k, n)-F d^{(2)}(k, n)=F d^{(3)}(k, n)-F d^{(1)}(k, n) .
$$

Theorem 4. Let $k \geq 2, n \geq 1,1 \leq r \leq k-2$ be integers. Then

$$
\begin{aligned}
& \text { (i) } \sigma_{-r_{0}}(k, n)=\sigma_{+r_{0}}(k, n)=F d^{(1)}(k, n), \\
& \text { (ii) } \sigma_{0}(k, n)=F d^{(2)}(k, n), \\
& \text { (iii) } \sigma(k, n)=F d^{(3)}(k, n) .
\end{aligned}
$$

Proof. The equality (i) follows immediately by Theorem 1 and (4).

We shall show that $\sigma_{0}(k, n)=F d^{(2)}(k, n)$. If $n=1,2, \ldots$, $k-2$, then there is no $k$-decomposition of the number $n$ into parts $k-1$ and $k$. So $\sigma_{0}(k, n)=0=F d^{(2)}(k, n)$. If $n=k-$ $1, k$, then there is a unique $k$-decomposition of the number $n$; hence $\sigma_{0}(k, n)=1=F d^{(2)}(k, n)$. Let $n \geq k+1$. Assume that the equality holds for an arbitrary $t<n$. We shall show that $\sigma_{0}(k, n)=F d^{(2)}(k, n)$. Let $n=n_{1}+n_{2}+\cdots+n_{p}$ be a $k$-decomposition of the number $n$ into parts $k$ and $k-1$. If $n_{p}=k$, then $n=n_{1}+n_{2}+\ldots+n_{p-1}+k$ so $n-k=n_{1}+$ $n_{2}+\cdots+n_{p-1}$. By induction's hypothesis there are $F d^{(2)}(k, n-$ $k$ ) $k$-decompositions in this case. If $n_{p}=k-1$ then proving analogously we obtain $F d^{(2)}(k, n-k+1) k$-decompositions 
of the form $n=n_{1}+n_{2}+\cdots+n_{p-1}+k-1$. From the above we have $F d^{(2)}(k, n-k)+F d^{(2)}(k, n-k+1) k$-decompositions of the number $n$ into parts $k$ and $k-1$, and by the definition of $F d^{(2)}(k, n)$ it follows that $\sigma_{0}(k, n)=F d^{(2)}(k, n)$.

Now we shall prove that $\sigma(k, n)=F d^{(3)}(k, n)$. From the definition of $\sigma(k, n)$ we obtain that

$$
\begin{aligned}
\sigma(k, n)= & \sum_{j=-(k-2)}^{k-2} \sigma_{j}(k, n) \\
= & 2 \sum_{r=1}^{k-2} \sigma_{+r}(k, n)+\sigma_{0}(k, n) \\
= & F d^{(1)}(k, n) \\
& +\sum_{r=1}^{k-2} \sigma_{+r}(k, n)+\sigma_{0}(k, n)-\sigma_{0}(k, n) \\
= & 2 F d^{(1)}(k, n)-F d^{(2)}(k, n),
\end{aligned}
$$

by the statements (i) and (ii) of this theorem.

Then the statement (iii) follows immediately by Lemma 2 , which ends the proof.

Theorem 5. Let $k \geq 2, n \geq k-1$ and $1 \leq r \leq k-2$ be integers. Then

$$
\sigma_{-r}(k, p k+i)=2^{p},
$$

for natural $p$ and $i=1,2, \ldots, k-(p+2)$.

Proof. Let $p$ be natural and $i=1,2, \ldots, k-(p+2)$. The number $p k+i$ is equal to $(i+l)+l \cdot(k-1)+(p-l) \cdot k$, for $l=$ $0,1, \ldots, p$, and all $r_{-} k$-decompositions of $p k+i$ have the form $(i+l)+n_{1}+\ldots+n_{p}$. We can put $k-1$ on $l$ positions, so we have $\left(\begin{array}{l}p \\ l\end{array}\right)$ possibilities. The sum $\sum_{l=0}^{p}\left(\begin{array}{l}p \\ l\end{array}\right)$ is equal to $2^{p}$ which ends the proof.

Now we give applications of distance Fibonacci numbers for counting of the number of other special decompositions of the number $n$.

Let $k \geq 2, n \geq k$ be integers and let $\sigma_{ \pm r_{0}}(k, n)$ be the numbers of all decomposition of the number $n=n_{1}+n_{2}+\cdots+n_{p}$, where $n_{i} \in\{k, k-1\}$, for $i=2, \ldots, p-1$ and $n_{1}, n_{p} \in\{1, \ldots, k\}$.

Theorem 6. Let $k \geq 2, n \geq k$ be integers. Then

$$
\sigma_{ \pm r_{0}}(k, n)=\sum_{r=1}^{k} F d^{(1)}(k, n-r) .
$$

Proof. Let $n=n_{1}+n_{2}+\cdots+n_{p}$ be a decomposition of the number $n$, where $n_{i} \in\{k, k-1\}$, for $i=2, \ldots, p-1$ and $n_{1}, n_{p} \in$ $\{1, \ldots, k\}$. Then $n-n_{p}=n_{1}+n_{2}+\cdots+n_{p-1}$, where $n_{1}+n_{2}+$ $\cdots+n_{p-1}$ is either a $r_{-} k$-decomposition or a $k$-decomposition of the number $n-n_{p}$. Since $n_{p} \in\{1, \ldots, k\}$ by Theorem $4(\mathrm{i})$ it follows that $\sigma_{ \pm r_{0}}(k, n)=\sum_{r=1}^{k} F d^{(1)}(k, n-r)$, which ends the proof.
Theorem 7. Let $k \geq 3, n \geq k$ be integers. Then

$$
\begin{aligned}
& \sum_{i=1}^{k-2} \sum_{j=1}^{k-2} F d^{(2)}(k, n-(i+j)) \\
&=\sum_{i=1}^{k-2}\left(F d^{(1)}(k, n-i)-F d^{(2)}(k, n-i)\right),
\end{aligned}
$$

for natural $p$ and $i=1,2, \ldots, k-(p+2)$.

Proof. Let $k \geq 2, n \geq k$ be integers. Let $n=n_{1}+n_{2}+\cdots+n_{p}$ be a decomposition $\eta$ of the number $n$, where $n_{i} \in\{k, k-$ $1\}$, for $i=2, \ldots, p-1$ and $n_{1}, n_{p} \in\{1, \ldots, k-2\}$. Then the number of such decomposition is equal to the number of $k$ decomposition of the number $n-\left(n_{1}+n_{p}\right)$. By Theorem 4(ii) we obtain that we have $F d^{(2)}\left(k, n-\left(n_{1}+n_{p}\right)\right)$ decompositions $\eta$. Since $n_{1}, n_{p} \in\{1, \ldots, k-2\}$ it is clear that totally we have

$$
\sum_{i=1}^{k-2} \sum_{j=1}^{k-2} F d^{(2)}(k, n-(i+j))
$$

decompositions of the number $n$.

On the other hand, we have that the total number of decompositions $\eta$ is equal to the number of $r_{-} k$-decompositions of the number $n-n_{p}$. Since $n_{p} \in\{1, \ldots, k-2\}$ by formula (5) and Theorem 4 we obtain

$$
\sum_{i=1}^{k-2}\left(F d^{(1)}(k, n-i)-F d^{(2)}(k, n-i)\right) .
$$

From the above it immediately follows that

$$
\begin{aligned}
& \sum_{i=1}^{k-2} \sum_{j=1}^{k-2} F d^{(2)}(n-(i+j)) \\
& =\sum_{i=1}^{k-2}\left(F d^{(1)}(k, n-i)-F d^{(2)}(k, n-i)\right),
\end{aligned}
$$

which ends the proof.

\section{Identities for $F d^{(1)}(k, n)$, $F d^{(2)}(k, n)$, and $F d^{(3)}(k, n)$}

In this section we give some identities for distance Fibonacci numbers: $F d^{(i)}(k, n)$, for $i=1,2,3$.

Theorem 8. Let $k \geq 2, n \geq k$ be integers. Then

$$
\begin{aligned}
& \sum_{j=1}^{3} a_{j} F d^{(j)}(k, n) \\
&=\sum_{i=0}^{p}\left(\begin{array}{c}
p \\
i
\end{array}\right)\left(\sum_{j=1}^{3} a_{j} F d^{(j)}(k, n-p k+i)\right) .
\end{aligned}
$$


Proof (by induction on $n$ ). For $n=k$ we have $p=1$ and

$$
\begin{aligned}
& \sum_{j=1}^{3} a_{j} F d^{(j)}(k, k) \\
& \quad=\sum_{j=1}^{3} a_{j}\left(F d^{(j)}(k, k-k)+F d^{(j)}(k, k-k+1)\right) \\
& =\sum_{j=1}^{3} a_{j}\left(F d^{(j)}(k, 0)+F d^{(j)}(k, 1)\right) \\
& =\sum_{j=1}^{3} a_{j}\left(F d^{(j)}(k, k-1 \cdot k+0)\right. \\
& =\sum_{i=0}^{1}\left(\begin{array}{l}
1 \\
i
\end{array}\right) \sum_{j=1}^{3} a_{j} F d^{(j)}(k, i) .
\end{aligned}
$$

Let $n>k$. Assume that (17) is true for arbitrary $t<n$ and we prove it for $n$. Using induction's assumption for $t=n-k$ and $t=n-k+1$ we have

$$
\begin{aligned}
F d^{(j)} & (k, n) \\
= & F d^{(j)}(k, n-k)+F d^{(j)}(k, n-k+1) \\
= & \sum_{i=0}^{p}\left(\begin{array}{c}
p \\
i
\end{array}\right) F d^{(j)}(k, n-k-p k+i) \\
& +\sum_{i=0}^{p}\left(\begin{array}{c}
p \\
i
\end{array}\right) F d^{(j)}(k, n-k+1-p k+i) \\
= & \sum_{i=0}^{p}\left(\begin{array}{c}
p \\
i
\end{array}\right)\left[F d^{(j)}(k, n-k-p k+i)\right. \\
= & \sum_{i=0}^{p}\left(\begin{array}{c}
p \\
i
\end{array}\right)\left[F d^{(j)}(k, n-k+1-p k+i)\right] \\
= & \sum_{i=0}^{p}\left(\begin{array}{c}
p \\
i
\end{array}\right) F d^{(j)}(k, n-p k+i),
\end{aligned}
$$

which ends the proof.

Corollary 9. Let $k \geq 2, n \geq k$ be integers. Then

(iv) $F d^{(1)}(k, n)=\sum_{i=0}^{p}\left(\begin{array}{c}p \\ i\end{array}\right) F d^{(1)}(k, n-p k+i)$,

(v) $F d^{(2)}(k, n)=\sum_{i=0}^{p}\left(\begin{array}{c}p \\ i\end{array}\right) F d^{(2)}(k, n-p k+i)$,

(vi) $F d^{(3)}(k, n)=\sum_{i=0}^{p}\left(\begin{array}{c}p \\ i\end{array}\right) F d^{(3)}(k, n-p k+i)$.
Proof. (iv) This formula directly follows from Theorem 8 putting $a_{1}=1$ and $a_{2}=a_{3}=0$. For (v) and (vi) analogously.

Theorem 10. Let $k \geq 3, n \geq k$ be integers. Then

$$
F d^{(1)}(k, n)=\sum_{i=0}^{k-2} F d^{(2)}(k, n-i)
$$

Proof (by induction on $n$ ). For $n=k$ we have $F d^{(1)}(k, k)=$ $F d^{(1)}(k, 0)+F d^{(1)}(k, 1)=1+1=2$. The right side of $(20)$ has the form $\sum_{i=0}^{k-2} F d^{(2)}(k, k-i)=F d^{(2)}(k, k-0)+F d^{(2)}(k, k-$ $1)+F d^{(2)}(k, k-2)+\cdots+F d^{(2)}(k, k-(k-2))=F d^{(2)}(k, k)+$ $F d^{(2)}(k, k-1)+F d^{(2)}(k, k-2)+\cdots+F d^{(2)}(k, 2)=1+1+0+$ $\cdots+0=2$.

Let $n>k$. Assume that (20) is true for arbitrary $t<n$ and we prove it for $n$. Using induction's assumption for $t=n-k$ and $t=n-k+1$ we have

$$
\begin{aligned}
& F d^{(1)}(k, n) \\
&= F d^{(1)}(k, n-k)+F d^{(1)}(k, n-k+1) \\
&= \sum_{i=0}^{k-2} F d^{(2)}(k, n-k-i) \\
&+\sum_{i=0}^{k-2} F d^{(2)}(k, n-k+1-i) \\
&= \sum_{i=0}^{k-2}\left[F d^{(2)}(k, n-k-i)\right. \\
&= \sum_{i=0}^{k-2}\left[F d^{(2)}(k, n-i-k)\right. \\
&= \sum_{i=0}^{k-2} F d^{(2)}(k, n-i), \\
&\left.+F d^{(2)}(k, n-i-k+1)\right]
\end{aligned}
$$

which ends the proof.

\section{Matrix Generators and Combinatorial Formulas for $F d^{(1)}(k, n)$, $F d^{(2)}(k, n)$, and $F d^{(3)}(k, n)$}

Matrix methods are important in recurrence relations. In the last decades some mathematicians have studied to find miscellaneous affinities between matrices and linear recurrences. Using matrix methods different identities and algebraic representations of considered sequences can be obtained, for instance $[1,2,4-6]$. Theory of Fibonacci numbers was previously complemented by the theory of so-called the Fibonacci Q-matrix or the golden matrix. It is worth mentioning that 
the American mathematician V. Hoggat was one of the first mathematicians who paid the attention to the Q-matrix. For the classical Fibonacci sequence the matrix generators, named as the golden matrix, have the form $Q=\left[\begin{array}{ll}1 & 1 \\ 1 & 0\end{array}\right]$ and $Q^{n}=\left[\begin{array}{cc}F_{n+1} & F_{n} \\ F_{n} & F_{n-1}\end{array}\right]$, for $n \geq 1$.

Golden number and golden section have many interesting applications in different areas of science (physics, chemistry, and mechanics); see for example $[7,8]$. In this section we give the matrix generators for distance Fibonacci numbers $F d^{(i)}(k, n)$, where $i=1,2,3$. The matrix generator of the distance Fibonacci numbers of the first kind $\operatorname{Fd}^{(1)}(k, n)$ was introduced in [1] and in this paper we apply this method for all kinds of distance Fibonacci numbers.

Let $k \geq 2$ be a fixed integer. Let $Q_{k}=\left[q_{t j}\right]$ be a square matrix of size $k$. For a fixed $1 \leq t \leq k$ an element $q_{t 1}$ is equal to the coefficient of $F d^{(i)}(k, n-t)$ in the recurrence formula for the distance Fibonacci numbers $\mathrm{Fd}^{(i)}(k, n), i=1,2,3$. For $j \geq 2$ we define $q_{t j}$ as follows:

$$
q_{t j}= \begin{cases}1 & \text { if } j=t+1 \\ 0 & \text { otherwise. }\end{cases}
$$

In other words,

$$
\begin{gathered}
Q_{2}=\left[\begin{array}{ll}
1 & 1 \\
1 & 0
\end{array}\right], \\
Q_{3}=\left[\begin{array}{lll}
0 & 1 & 0 \\
1 & 0 & 1 \\
1 & 0 & 0
\end{array}\right], \ldots, Q_{k}=\left[\begin{array}{ccccc}
0 & 1 & 0 & \cdots & 0 \\
0 & 0 & 1 & \cdots & 0 \\
\vdots & \vdots & \vdots & & \vdots \\
1 & 0 & 0 & \cdots & 1 \\
1 & 0 & 0 & \cdots & 0
\end{array}\right],
\end{gathered}
$$

and the matrix $Q_{k}$ is named as the distance Fibonacci matrix or the generator of the distance Fibonacci numbers $\mathrm{Fd}^{(i)}(k, n), i=1,2,3$.

For a fixed $1 \leq i \leq 3$ we define the square matrix $A_{k}^{(i)}$ of size $k$ named as the matrix of initial conditions of the form

$$
A_{k}^{(i)}=\left[\begin{array}{cccc}
F d^{(i)}(k, 2 k-2+u(i)) & F d^{(i)}(k, 2 k-3+u(i)) & \cdots & F d^{(i)}(k, k-1+u(i)) \\
F d^{(i)}(k, 2 k-3+u(i)) & F d^{(i)}(k, 2 k-4+u(i)) & \cdots & F d^{(i)}(k, k-2+u(i)) \\
\vdots & \vdots & \ddots & \vdots \\
F d^{(i)}(k, k+u(i)) & F d^{(i)}(k, k-1+u(i)) & \cdots & F d^{(i)}(k, 1+u(i)) \\
F d^{(i)}(k, k-1+u(i)) & F d^{(i)}(k, k-2+u(i)) & \cdots & F d^{(i)}(k, 0+u(i))
\end{array}\right]
$$

where

$$
u(i)= \begin{cases}i-1 & \text { for } i=1,2 \\ k-1 & \text { for } i=3\end{cases}
$$

Theorem 11. Let $k \geq 2, n \geq k$ be integer. Then for a fixed $1 \leq i \leq 3$ holds

$$
A_{k}^{(i)} Q_{k}^{n}=\left[\begin{array}{cccc}
F d^{(i)}(k, n+2 k-2+u(i)) & F d^{(i)}(k, n+2 k-3+u(i)) & \cdots & F d^{(i)}(k, n+k-1+u(i)) \\
F d^{(i)}(k, n+2 k-3+u(i)) & F d^{(i)}(k, n+2 k-4+u(i)) & \cdots & F d^{(i)}(k, n+k-2+u(i)) \\
\vdots & \vdots & \ddots & \vdots \\
F d^{(i)}(k, n+k+u(i)) & F d^{(i)}(k, n+k-1+u(i)) & \cdots & F d^{(i)}(k, n+1+u(i)) \\
F d^{(i)}(k, n+k-1+u(i)) & F d^{(i)}(k, n+k+u(i)) & \cdots & F d^{(i)}(k, n+0+u(i))
\end{array}\right] .
$$

Since the proof is analogous as in [1] we omit it. To obtain other matrix generators apart distance Fibonacci numbers $F d^{(i)}(k, n), i=1,2,3$, we define a collection of special sequences which are given by the same $k$ th order linear recurrence relations as $F d^{(i)}(k, n), i=1,2,3$. These sequences give auxiliary tools for other matrix generators of $F d^{(i)}(k, n), i=$ $1,2,3$ and their explicit formulas.
Let $k \geq 2, n \geq 0$ be integers. Let $\mathscr{F}_{k}=\left\{F d_{l}^{(4)}(k, n), l=0\right.$, $1, \ldots, k-1\}$, where $\left\{F d_{l}^{(4)}(k, n)\right\}$ is the sequence defined as follows:

$$
\begin{array}{r}
F d_{l}^{(4)}(k, n)=F d_{l}^{(4)}(k, n-k+1)+F d_{l}^{(4)}(k, n-k), \\
\text { for } n \geq k,
\end{array}
$$


with the initial conditions $F d_{l}^{(4)}(k, l)=1$ and $F d_{l}^{(4)}(k, j)=0$ for $j \neq l$.

The number $F d_{1}^{(4)}(k, n)$ will be also denoted shortly by $F d^{(4)}(k, n)$ and named as the $n$th distance Fibonacci number of the fourth kind. If $k=2$ and $n \geq 1$, then $F d^{(4)}(2, n)=F_{n-1}$.

By simple observation we obtain the following relations between numbers $F d_{l}^{(4)}(k, n)$, for $l=0,1, \ldots, k-1$ :

$$
\begin{gathered}
F d_{0}^{(4)}(k, n)=F d^{(4)}(k, n-k+1), \\
F d_{l}^{(4)}(k, n)=F d^{(4)}(k, n-l+1) \quad \text { for } 2 \leq l \leq k-1 .
\end{gathered}
$$

Using sequences from the collection $\mathscr{F}_{k}$ we can generate the distance Fibonacci numbers $F d^{(i)}(k, n), i=1,2,3$ :

$$
\begin{array}{r}
F d^{(1)}(k, n)=\sum_{l=0}^{k-1} F d^{(1)}(k, l) F d_{l}^{(4)}(k, n)=\sum_{l=0}^{k-1} F d_{l}^{(4)}(k, n), \\
F d^{(2)}(k, n)=\sum_{l=k-1}^{k} F d^{(2)}(k, l) F d_{l-1}^{(4)}(k, n-1) \\
=\sum_{l=k-1}^{k} F d_{l-1}^{(4)}(k, n-1) \quad \text { for } n \geq 1, \\
F d^{(3)}(k, n) \quad \text { for } n \geq k-1 .
\end{array}
$$

Theorem 12. Let $k \geq 2$ be integer. Then

(i) $F d^{(1)}(k, n)=\sum_{t=0}^{k-1} F d^{(4)}(k, n-t)$ for $n \geq k$,

(ii) $F d^{(2)}(k, n)=F d^{(4)}(k, n-(k-2))+F d^{(4)}(k, n-(k-1))$ for $n \geq k$,

(iii) $F d^{(3)}(k, n)=F d^{(4)}(k, n-2(k-1))+3 F d^{(4)}(k, n-(k-$ $1))+4 \sum_{t=2}^{k-2} F d^{(4)}(k, n-(k-1)-t+1)+3 F d^{(4)}(k, n-$ $2(k-1)+1)$ for $n \geq 2 k-1$.
Proof. Let $F d_{l}^{(1)}(k, n)$ denote a sequence defined by the same recurrence as $F d^{(1)}(k, n)$ with initial conditions

$$
F d_{l}^{(1)}(k, n)=\left\{\begin{array}{ll}
F d^{(1)}(k, l) & \text { if } n=l \\
0 & \text { if } n \neq l
\end{array} \quad \text { for } n \leq k-1 .\right.
$$

Then

$$
\begin{aligned}
F d_{l}^{(1)}(k, n) \\
=F d_{0}^{(1)}(k, n)+\cdots+F d_{k-1}^{(1)}(k, n) \\
=F d^{(1)}(k, 0) F d_{0}^{(1)}(k, n) \\
\quad+\cdots+F d^{(1)}(k,-1) F d_{k-1}^{(1)}(k, n) \\
=1 F d_{0}^{(1)}(k, n)+\cdots+1 F d_{k-1}^{(1)}(k, n) \\
=\sum_{t=0}^{k-1} F d^{(4)}(k, n-t) .
\end{aligned}
$$

We prove analogously formulas (ii) and (iii).

Using the above theorem we obtain a new matrix generator for distance Fibonacci numbers $F d^{(i)}(k, n), i=1,2,3$.

Corollary 13. For the distance Fibonacci numbers $\mathrm{Fd}^{(i)}(k, n)$, $i=1,2,3$, Theorem 12 gives its matrix generator, respectively:

(1) $\sum_{t=0}^{k-1} Q_{k}^{n-t}$ for $n \geq k$,

(2) $Q_{k}^{n-(k-2)}+Q_{k}^{n-(k-1)}$ for $n \geq k$,

(3) $Q_{k}^{n-2(k-1)}+3 Q_{k}^{n-(k-1)}+4 \sum_{t=2}^{k-2} Q_{k}^{n-(k-1)-t+1}+Q_{k}^{n-2(k-1)+1}$ for $n \geq 2 k-1$.

Theorem 14. Let $k \geq 2, n \geq k$ be integer. Then

$$
F d^{(4)}(k, n+1)=\sum_{\substack{k_{1}, k_{2} \\
k_{1} \cdot k+k_{2} \cdot(k-1)=n}}\left(\begin{array}{c}
k_{1}+k_{2} \\
k_{1}
\end{array}\right) .
$$

Proof. We consider a digraph $D_{k}$ represented by adjacency matrix $Q_{k}$ auxiliary (Figure 1).

Note that matrix $Q_{k}^{n}$ has the following form:

$$
\left[\begin{array}{cccc}
F d^{(4)}(k, n+1) & F d^{(4)}(k, n) & \cdots & F d^{(4)}(k, n-(k-2)) \\
F d^{(4)}(k, n+2) & F d^{(4)}(k, n+1) & \cdots & F d^{(4)}(k, n-(k-1)) \\
\vdots & \vdots & \ddots & \vdots \\
F d^{(4)}(k, n+(k-1)) & F d^{(4)}(k, n+(k-2)) & \cdots & F d^{(4)}(k, n) \\
F d^{(4)}(k, n) & F d^{(4)}(k, n-1) & \cdots & F d^{(4)}(k, n-(k-1))
\end{array}\right] .
$$

It is well known that $q_{i j} \in Q_{k}^{n}$ is equal to the number of all distinct paths of length $n$ between vertices $v_{i}$ and $v_{j}$ in the digraph $D_{k}$.
Each path $P$ from $v_{1}$ to $v_{1}$ in digraph $D_{k}$ has the following form: $P: v_{1} \rightarrow v_{x_{1}} \rightarrow v_{1} \rightarrow v_{x_{2}} \rightarrow v_{1} \cdots v_{1} \rightarrow v_{x_{i}} \rightarrow v_{1}$ where $v_{1} \rightarrow v_{x}$ denotes the unique shortest path from $v_{1}$ to $v_{x}$ in 
TABle 1: The $n$th distance Fibonacci numbers $F d^{(1)}(k, n)$.

\begin{tabular}{lllllllllllllcccc}
\hline$n$ & 0 & 1 & 2 & 3 & 4 & 5 & 6 & 7 & 8 & 9 & 10 & 11 & 12 & 13 \\
\hline$F d^{(1)}(2, n)$ & 1 & 1 & 2 & 3 & 5 & 8 & 13 & 21 & 34 & 55 & 89 & 144 & 233 & 377 & 610 \\
$F d^{(1)}(3, n)$ & 1 & 1 & 1 & 2 & 2 & 3 & 4 & 5 & 7 & 9 & 12 & 16 & 21 & 28 & 37 \\
$F d^{(1)}(4, n)$ & 1 & 1 & 1 & 1 & 2 & 2 & 2 & 3 & 4 & 4 & 5 & 7 & 8 & 9 & 12 \\
$F d^{(1)}(5, n)$ & 1 & 1 & 1 & 1 & 1 & 2 & 2 & 2 & 2 & 3 & 4 & 4 & 4 & 5 & 7 \\
$F d^{(1)}(6, n)$ & 1 & 1 & 1 & 1 & 1 & 1 & 2 & 2 & 2 & 2 & 2 & 3 & 4 & 4 & 4 \\
$F d^{(1)}(7, n)$ & 1 & 1 & 1 & 1 & 1 & 1 & 1 & 2 & 2 & 2 & 2 & 2 & 2 & 3 \\
$F d^{(1)}(8, n)$ & 1 & 1 & 1 & 1 & 1 & 1 & 1 & 1 & 2 & 2 & 2 & 2 & 2 & 2 \\
\hline
\end{tabular}

TABLE 2: The $n$th distance Fibonacci numbers $F d^{(2)}(k, n)$.

\begin{tabular}{lllllllllllllccc}
\hline$n$ & 0 & 1 & 2 & 3 & 4 & 5 & 6 & 7 & 8 & 9 & 10 & 11 & 12 & 13 \\
\hline$F d^{(2)}(2, n)$ & 0 & 1 & 2 & 3 & 5 & 8 & 13 & 21 & 34 & 55 & 89 & 144 & 233 & 377 & 610 \\
$F d^{(2)}(3, n)$ & 0 & 0 & 1 & 1 & 1 & 2 & 2 & 3 & 4 & 5 & 7 & 9 & 12 & 16 & 21 \\
$F d^{(2)}(4, n)$ & 0 & 0 & 0 & 1 & 1 & 0 & 1 & 2 & 1 & 1 & 3 & 3 & 2 & 4 & 6 \\
$F d^{(2)}(5, n)$ & 0 & 0 & 0 & 0 & 1 & 1 & 0 & 0 & 1 & 2 & 1 & 0 & 1 & 3 & 3 \\
$F d^{(2)}(6, n)$ & 0 & 0 & 0 & 0 & 0 & 1 & 1 & 0 & 0 & 0 & 1 & 2 & 1 & 0 & 0 \\
$F d^{(2)}(7, n)$ & 0 & 0 & 0 & 0 & 0 & 0 & 1 & 1 & 0 & 0 & 0 & 0 & 1 & 2 & 1 \\
$F d^{(2)}(8, n)$ & 0 & 0 & 0 & 0 & 0 & 0 & 0 & 1 & 1 & 0 & 0 & 0 & 0 & 0 \\
\hline
\end{tabular}

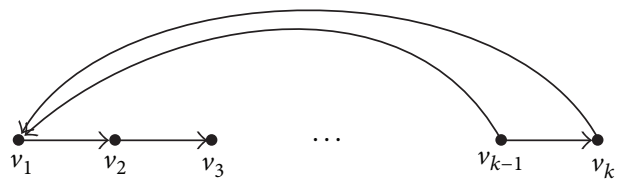

FIGURE 1: A digraph $D_{k}$.

digraph $D_{k}$. Parts $v_{1} \rightarrow v_{x} \rightarrow v_{1}$ are cycles of the length $x$, where $x$ is $k$ or $k-1$. Thus the length of the path $P$ is equal to $x_{1}+x_{2}+\cdots+x_{i}$. There exists one-to-one correspondence between the path $P$ and a tuple $\left(x_{1}, x_{2}, \ldots, x_{i}\right)$. If the path $P$ has a length $n$, then the corresponding tuple is a decomposition of an integer $n$ when $k$ occurs $k_{1}$ times and $k-1$ occurs $k_{2}$ times and $k_{1} \cdot k+k_{2} \cdot(k-1)=n$. The number of such tuples is equal to the binomial coefficient $\left(\begin{array}{c}k_{1}+k_{2} \\ k_{1}\end{array}\right)$. We determine analogously the number of different paths between other pairs of vertices.

Using Theorems 14 and 12 we can prove the following.

Theorem 15. Let $k \geq 2$ be integer. Then

$$
\begin{aligned}
& F d^{(1)}(k, n) \\
& =\sum_{i=0}^{k-1} \sum_{\substack{k_{1}, k_{2} \\
k_{1} \cdot k+k_{2} \cdot(k-1)=n-i-1}}\left(\begin{array}{c}
k_{1}+k_{2} \\
k_{1}
\end{array}\right), \\
& n \geq k,
\end{aligned}
$$

$$
\begin{aligned}
& F^{(2)}(k, n) \\
& =\sum_{i=k-2}^{k-1} \sum_{\substack{k_{1}, k_{2} \\
k_{1} \cdot k+k_{2} \cdot(k-1)=n-i-1}}\left(\begin{array}{c}
k_{1}+k_{2} \\
k_{1}
\end{array}\right),
\end{aligned}
$$$$
n \geq k \text {, }
$$

$$
\begin{aligned}
& F d^{(3)}(k, n) \sum_{k_{1} \cdot k+k_{2} \cdot(k-1)=n-1-2(k-1)}\left(\begin{array}{c}
k_{1}+k_{2} \\
k_{1}
\end{array}\right) \\
& +3 \sum_{\substack{k_{1}, k_{2}, k_{2} \\
k_{1} \cdot k+k_{2} \cdot(k-1)=n-1-(k-1)}}\left(\begin{array}{c}
k_{1}+k_{2} \\
k_{1}
\end{array}\right) \\
& +4 \sum_{i=2}^{k-2} \sum_{\substack{k_{1}, k_{2} \\
k_{1} \cdot k+k_{2} \cdot(k-1)=n-i-(k-1)}}\left(\begin{array}{c}
k_{1}+k_{2} \\
k_{1}
\end{array}\right) \\
& \sum_{\substack{k_{1}, k_{2} \\
k_{1} \cdot k+k_{2} \cdot(k-1)=n-2(k-1)}}\left(\begin{array}{c}
k_{1}+k_{2} \\
k_{1}
\end{array}\right), \\
& n \geq 2 k-1 .
\end{aligned}
$$

Tables 1, 2, 3, and 4 present first words of four types of distance Fibonacci numbers $F d^{(1)}(k, n), F^{(2)}(k, n), F d^{(3)}(k, n)$, and $F d^{(4)}(k, n)$. 
TABle 3: The $n$th distance Fibonacci numbers $F d^{(3)}(k, n)$.

\begin{tabular}{llllllllllllllcc}
\hline$n$ & 0 & 1 & 2 & 3 & 4 & 5 & 6 & 7 & 8 & 9 & 10 & 11 & 12 & 13 & 14 \\
\hline$F d^{(3)}(2, n)$ & 1 & 1 & 2 & 3 & 5 & 8 & 13 & 21 & 34 & 55 & 89 & 144 & 233 & 377 & 610 \\
$F d^{(3)}(3, n)$ & 1 & 1 & 1 & 3 & 3 & 4 & 6 & 7 & 10 & 13 & 17 & 23 & 30 & 40 & 53 \\
$F d^{(3)}(4, n)$ & 1 & 1 & 1 & 1 & 3 & 4 & 3 & 4 & 7 & 7 & 7 & 11 & 14 & 14 & 18 \\
$F d^{(3)}(5, n)$ & 1 & 1 & 1 & 1 & 1 & 3 & 4 & 4 & 3 & 4 & 7 & 8 & 7 & 7 & 11 \\
$F d^{(3)}(6, n)$ & 1 & 1 & 1 & 1 & 1 & 1 & 3 & 4 & 4 & 4 & 3 & 4 & 7 & 8 & 8 \\
$F d^{(3)}(7, n)$ & 1 & 1 & 1 & 1 & 1 & 1 & 1 & 3 & 4 & 4 & 4 & 4 & 3 & 4 \\
$F d^{(3)}(8, n)$ & 1 & 1 & 1 & 1 & 1 & 1 & 1 & 1 & 3 & 4 & 4 & 4 & 4 & 4 \\
\hline
\end{tabular}

TABle 4: The $n$th distance Fibonacci numbers $F d^{(4)}(k, n)$.

\begin{tabular}{llllllllllllccccc}
\hline$n$ & 0 & 1 & 2 & 3 & 4 & 5 & 6 & 7 & 8 & 9 & 10 & 11 & 12 & 13 \\
\hline$F d^{(4)}(2, n)$ & 0 & 1 & 1 & 2 & 3 & 5 & 8 & 13 & 21 & 34 & 55 & 89 & 144 & 233 & 377 \\
$F d^{(4)}(3, n)$ & 0 & 1 & 0 & 1 & 1 & 1 & 2 & 2 & 3 & 4 & 5 & 7 & 9 & 12 & 16 \\
$F d^{(4)}(4, n)$ & 0 & 1 & 0 & 0 & 1 & 1 & 0 & 1 & 2 & 1 & 1 & 3 & 3 & 2 & 4 \\
$F d^{(4)}(5, n)$ & 0 & 1 & 0 & 0 & 0 & 1 & 1 & 0 & 0 & 1 & 2 & 1 & 0 & 1 & 3 \\
$F d^{(4)}(6, n)$ & 0 & 1 & 0 & 0 & 0 & 0 & 1 & 1 & 0 & 0 & 0 & 1 & 2 & 1 & 0 \\
$F d^{(4)}(7, n)$ & 0 & 1 & 0 & 0 & 0 & 0 & 0 & 1 & 1 & 0 & 0 & 0 & 0 & 1 \\
$F d^{(4)}(8, n)$ & 0 & 1 & 0 & 0 & 0 & 0 & 0 & 0 & 1 & 1 & 0 & 0 & 0 & 0 \\
\hline
\end{tabular}

\section{Conflict of Interests}

The authors declare that there is no conflict of interests regarding the publication of this paper.

\section{Acknowledgment}

The authors would like to thank the referee for helpful valuable suggestions which resulted in improvements to this paper.

\section{References}

[1] U. Bednarz, A. Włoch, and M. Wołowiec-Musiał, "Distance Fibonacci numbers, their interpretations and matrix generators," Commentationes Mathematicae, vol. 53, no. 1, pp. 35-46, 2013.

[2] E. Kiliç, "The generalized order- $k$ Fibonacci-Pell sequence by matrix methods," Journal of Computational and Applied Mathematics, vol. 209, no. 2, pp. 133-145, 2007.

[3] I. Włoch and A. Włoch, "Generalized sequences and $k$ independent sets in graphs," Discrete Applied Mathematics, vol. 158, no. 17, pp. 1966-1970, 2010.

[4] J. Ercolano, "Matrix generators of Pell sequences," The Fibonacci Quarterly, vol. 17, no. 1, pp. 71-77, 1979.

[5] E. Kilic and D. Tasci, "On the generalized Fibonacci and Pell sequences by Hessenberg matrices," Ars Combinatoria, vol. 94, pp. 161-174, 2010.

[6] A. Włoch and M. Wołowiec-Musiał, "Generalized Pell numbers and some relations with Fibonacci numbers," Ars Combinatoria, vol. 109, pp. 391-403, 2013.

[7] D. Jakubczyk, "The exact results in the one-dimensional attractive Hubbard model," Acta Physica Polonica B, vol. 45, no. 8, pp. 1759-1770, 2014.

[8] M. Startek, A. Włoch, and I. Włoch, "Fibonacci numbers and Lucas numbers in graphs," Discrete Applied Mathematics, vol. 157, no. 4, pp. 864-868, 2009. 


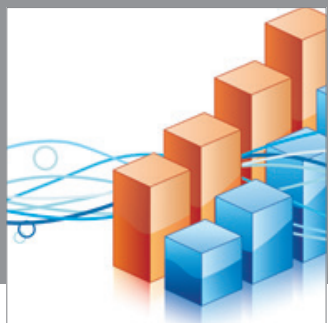

Advances in

Operations Research

mansans

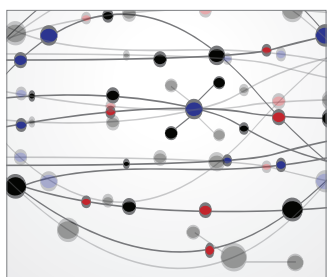

The Scientific World Journal
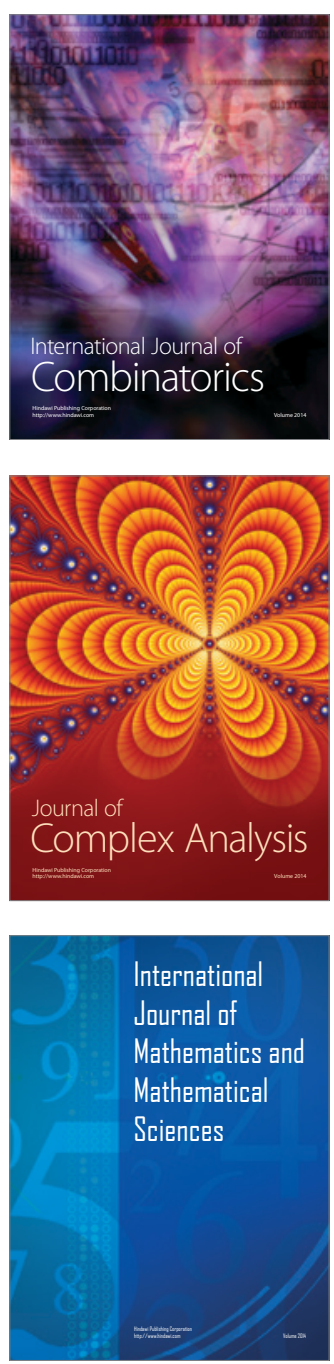
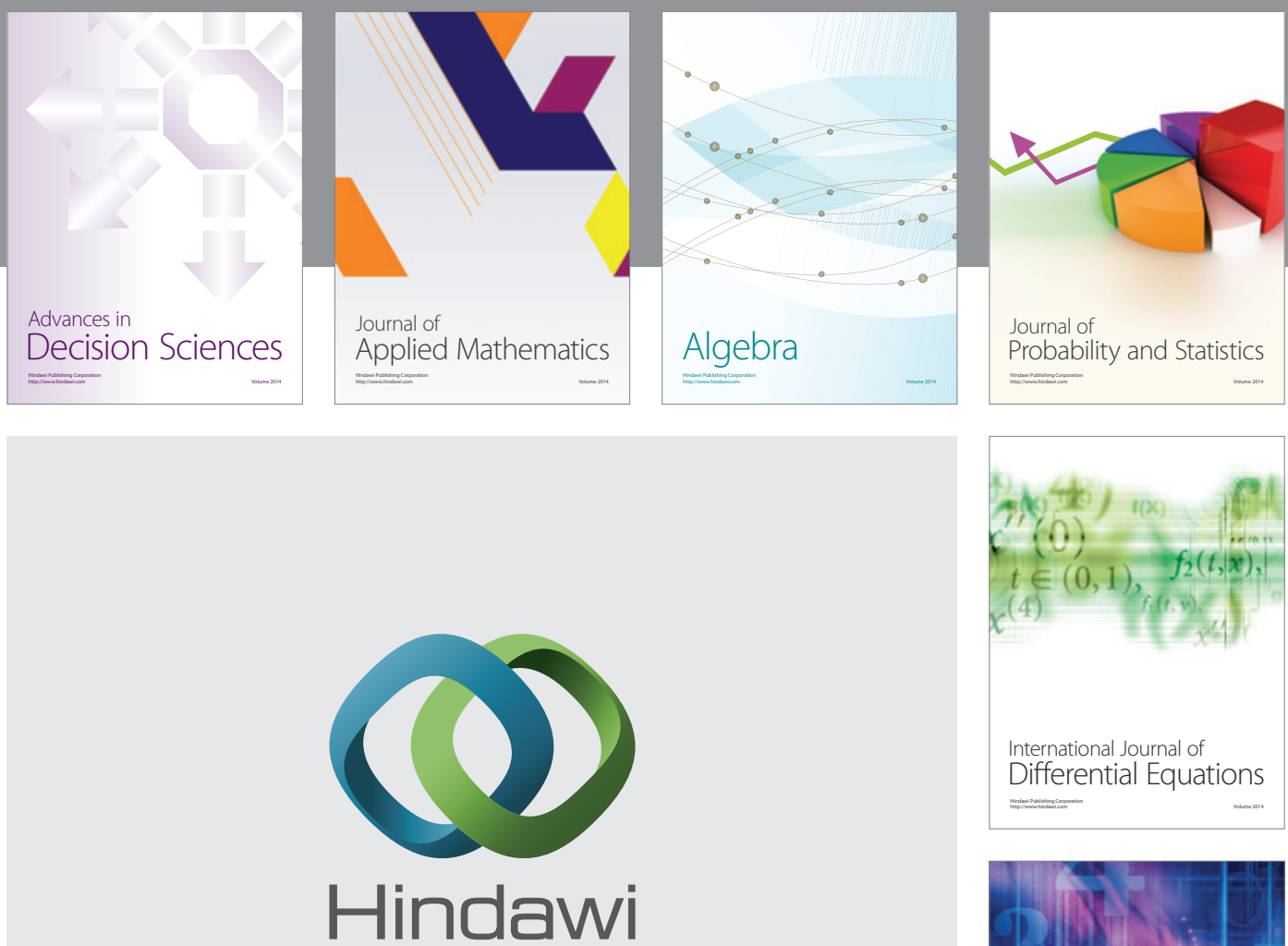

Submit your manuscripts at http://www.hindawi.com
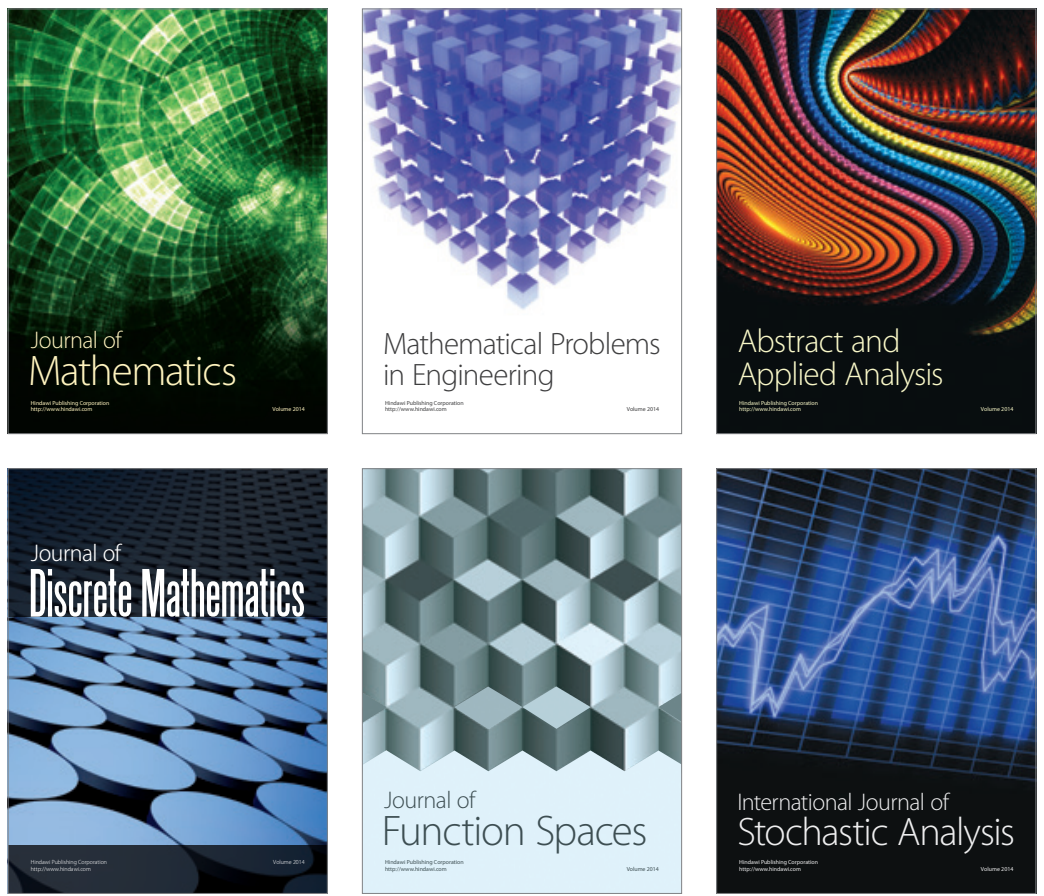

Journal of

Function Spaces

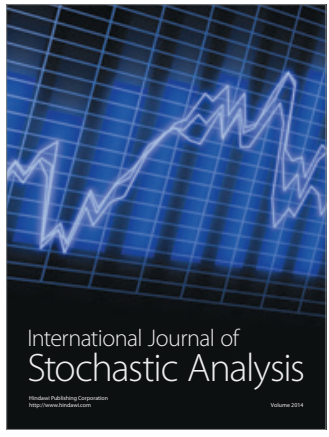

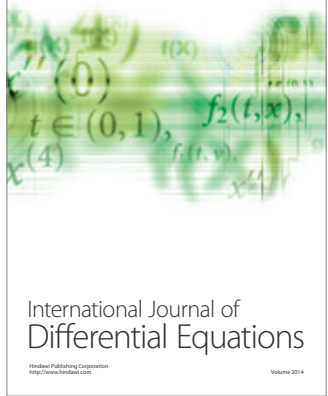
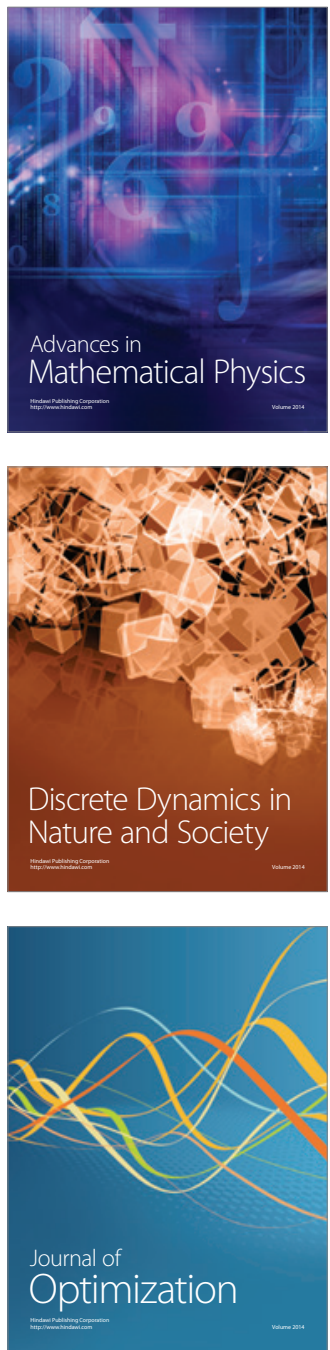